\title{
Contact Angle Measurement for The Surface Characterization of Solids
}

\author{
D. P. Subedi \\ Department of Natural Sciences, School of Science, Kathmandu University, Dhulikhel, Kavre. \\ deepaksubedi2001@yahoo.com
}

\begin{abstract}
This paper reports a description of the theory of contact angle measurement and its use for the determination of surface energy of solids. The main objective of the work is to present a convenient approach to explain wetting phenomenon using contact angle theory. A brief review of Young's equation and Young-Dupre equation is also presented followed by an extended Fowke's equation widely used for the calculation of surface energy of solids. The two liquid model has been applied to determine the surface energy of polycarbonate and low density polyethylene.
\end{abstract}

Key words: Contact angle, surface energy, surface characterization, wettability, Young's equation, Fowke's model

\section{Introduction}

Over the years a large number of techniques have been developed to probe different aspects of the physics and chemistry of surfaces, however, only a few have found wide application in basic surface science and applied surface analysis [1]. The choice of the technique depends upon the type of the characterization to be made. Among the most widely used methods are X-ray photoelectron spectroscopy (XPS) and Fourier transform infrared spectroscopy (FTIR) are used to study the surface chemical composition. Similarly, scanning electron microscopy (SEM) and atomic force microscopy (AFM) are used to investigate the surface morphology of the material in atomic scale. These methods require relatively expensive equipments, skilled technicians and sophisticated techniques to interpret data. [2-3]. Measurement of surface energy of the solid can also provide a good understanding of the surface properties of a solid using relatively a very simple approach. The surface energy of a solid can be determined from the measurement of contact angle of a pure liquid drop on that solid. Contact angle measurement has been used in the study of surface energy, wettability and adhesion of low surface energy materials. In this paper, the theory of contact angle measurement and an important model for the determination of surface energy will be discussed.

\section{Theory of Contact Angle Measurement}

Studies concerning the interaction between a solid and liquid require reliable experimental values of contact angle and surface energy. The quantitative evaluation of the wetting of a solid by a liquid is made in terms of the contact angle $\theta$. Fig. 1 shows the schematic diagram for the contact angle and the interfacial tensions at the three phase boundary.

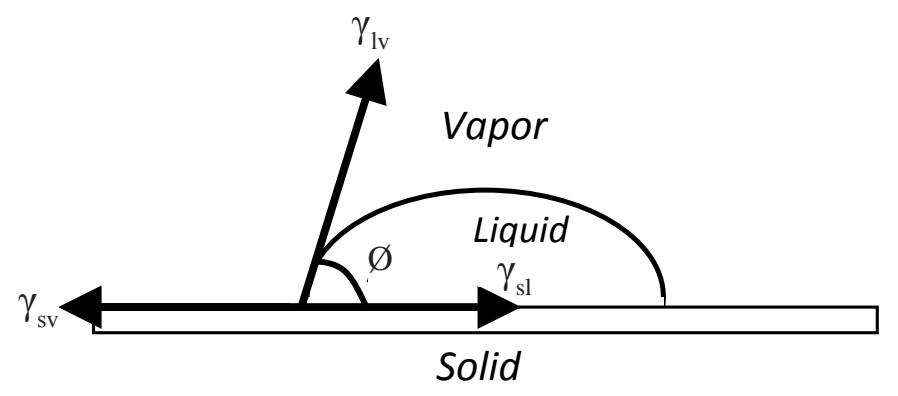

Fig. 1. Schematic diagram of the contact angle and interfacial tensions of the three surfaces at the three-phase boundary

The most important relation regarding the contact angle is the Young's equation that relates the contact angle $\theta$, liquid surface tension $\gamma_{\mathrm{l}}$, solid surface energy $\gamma_{\mathrm{s}}$, solid-liquid surface tension $\gamma_{\mathrm{sl}}$ as expressed in Eq.(1) [4]. 


$$
\gamma_{1} \cos \theta=\theta_{s} \gamma \gamma_{s l}
$$

For the convenience of understanding the wetting phenomenon, Eq. (1) can be rearranged as shown in Eq. (2).

$$
\cos \theta-\frac{\gamma_{s}-\gamma_{s l}}{\gamma_{I}}
$$

From Eq. (2), the value of $\cos \theta$ is determined by the relative magnitude of the three quantities namely, the surface tension of the liquid $\left(\gamma_{1}\right)$, surface energy of the solid $\left(\gamma_{s}\right)$ and the interfacial tension between the solid and the liquid $\left(\gamma_{\mathrm{s}}\right)$. A perfect wetting means the maximum value of $\cos \theta$ which is achieved when the contact angle is zero. There are two possibilities to inrease the value of $\cos \theta$, either by reducing the surface tension of the liquid or by increasing the surface energy of the solid. For example, we consider the case of contact angle of water (surface tension of 72.8 dynes/ $\mathrm{cm}$ at $25^{\circ} \mathrm{C}$ ) on a polyethylene which has a surface free energy of about $25 \mathrm{~mJ} / \mathrm{m}^{2}$. The contact angle of water on this solid is about $90^{\circ}$. In this case, the liquid will not wet the solid and we say that the surface is hydrophobic and has a poor wettability. If the surface tension of water is lowered by mixing surfactant in it keeping the solid surface unchanged, the contact angle will decrease i.e. water spreads on the surface of the solid. This phenomenon is very common in practice such as; the cleaning of oily dishes by soapwater and use of detergent in washing clothes. Mixing detergents to spray pesticides uniformly over the surface of plant leaves is another common example.

It is also possible to modify the solid- surface by grafting of hydrophilic functional groups to reduce the contact angle with pure water drop. We can interpret the wetting phenomena in terms of the force of cohesion and adhesion. For a high surface tension liquid drop placed on a solid surface of low surface energy, the force of attraction between the liquid molecules is stronger than the force of attraction between the liquid molecules and the atoms in the solid and hence the liquid forms a drop and there is a definite value of contact angle. However, if the surface tension of the liquid is lowered or the surface free energy of the solid is increased, the force of attraction between the liquid molecules and the atoms in the solid becomes more than the force of attraction between the liquid molecules and hence the liquids starts to spread on the solid surface. In other words, the lower the surface tension of the liquid or the higher the surface energy of the solid, better will be the wetting process.

\section{Calculation of Surface Energy}

Another important contribution in the field of surface analysis was made by Dupre [5]. According to this equation, work of adhesion $\mathrm{W}_{\mathrm{A}}$ between a solid (s) and a liquid (1) can be expressed as,

$$
\mathrm{W}_{\mathrm{A}}=\gamma_{\mathrm{s}}+\gamma_{\mathrm{I}}-\gamma_{\mathrm{sl}}
$$

This work of adhesion $\mathrm{W}_{\mathrm{A}}$ is the reversible work done in separation of unit area of solid/liquid interface and equals to one half of the work of cohesion when the arrangement of molecules in the surface region is the same as the bulk. Combining equation (1) and (3), gives the Young-Dupre equation which is represented by Eq. (4).

$$
\mathrm{W}_{\mathrm{A}}=\gamma_{\mathrm{s}}(\cos \theta+1)
$$

The work of adhesion $\mathrm{W}_{\mathrm{A}}$ can be evaluated from Eq. (4) using accessible quantities $\gamma_{1}$ and $\theta$. Equation (4) is the starting point for interpretation of all contact angle data. After the evaluation of $\mathrm{W}_{\mathrm{A}}$, the surface energy of the solid $\gamma_{\mathrm{s}}$ can be determined by further use of other models.

\section{Fowke's equation}

Fowke [6] proposed an approach to the problem of separating the surface energy of solid into its components. According to this approach, the total surface energy of a solid is expressed as the sum of the various components as expressed in Eq. (5),

$$
\gamma=\gamma^{d}+\gamma^{p}+\gamma^{\text {ind }}+\gamma^{H}
$$

where, the subscripts $d, p$, ind and $H$ refer to dispersion, polar, induced and hydrogen bond forces respectively. Finally it was suggested that the equation can be expressed with only the two dominant terms,

$$
\begin{aligned}
& \gamma=\gamma^{d}+\gamma^{H} \\
& \gamma^{A B}=\gamma^{p}+\gamma^{\text {ind }}+\gamma^{H}
\end{aligned}
$$

Where $\mathrm{AB}$ refers to the Acid- base interaction.

In order to calculate only the total surface energy, it is enough to know the contact angle with only one test liquid. But, if we would like to determine not only the total surface energy but also its associated polar and dispersion components we must measure 
contact angles with at least two test liquids. If we want to know the components related to acid base interaction, it is necessary to measure the contact angle with three test liquids. Therefore, in practice of surface energy calculation, one liquid, two liquid and three liquid models are used depending upon the required depth of the analysis. In this paer we will present only the two liquid model which is the most widely used method.

The two liquid model is also known as Owens-Wendt-Kaelble [7] model and sometimes termed as extended Fowke's model. The theoretical basis of this approach is the Young-Dupre equation as represented by Eq. 4. In this model, the work of adhesion $\mathrm{W}_{\mathrm{A}}$ is expressed in terms of the polar and dispersion components of the surface energy i.e.

$W_{A}=2\left[\left(\gamma_{1}{ }^{d}\right)\left(\gamma_{s}{ }^{d}\right)\right]^{\frac{1}{2}}+2\left[\left(\gamma_{1}{ }^{p}\right)\left(\gamma_{s}^{p}\right)\right]^{\frac{1}{2}}$

Where, $\gamma_{1}^{\mathrm{d}}$ and $\gamma_{1}^{\mathrm{p}}$ are the dispersion and polar components of the surface tension of the test liquid. Similarly, $\gamma_{s}^{d}$ and $\gamma_{s}^{p}$ are the dispersion and polar components of the surface energy of the solid. In actual measurement of contact angle and surface energy, $\gamma_{1}^{\mathrm{d}}$ and $\gamma_{1}^{\mathrm{p}}$ are known and the contact angle is measured to determine the value of $\gamma_{s}^{d}$ and $\gamma_{s}^{p}$. Since there are two unknown quantities, we need to measure the contact angle with two liquids in order to have to independent equations. For this purpose, one polar and one nonpolar liquid are used as test liquids.

Combining Eq. 4 and Eq. 8 we get

$\gamma_{1}(1+\cos \theta)=2\left[\left(\gamma_{1}^{d}\right)\left(\gamma_{s}^{d}\right)\right]^{\frac{1}{2}}+2\left[\left(\gamma_{1}^{p}\right)\left(\gamma_{s}^{p}\right)\right]^{\frac{1}{2}}$

For two liquids i and j, Eq. 9 can be written separately as,

$\gamma_{l i}\left(1+\cos \theta_{i}\right)=2\left[\left(\gamma_{l i}{ }^{d}\right)\left(\gamma_{s}{ }^{d}\right)\right]^{\frac{1}{2}}+2\left[\left(\gamma_{l i}{ }^{p}\right)\left(\gamma_{s}{ }^{p}\right)\right]^{\frac{1}{2}}$

and

$\gamma_{\mathrm{lj}}(1+\cos \theta j)=2\left[\left(\gamma_{\mathrm{lj}}{ }^{\mathrm{d}}\right)\left(\gamma_{\mathrm{s}}{ }^{\mathrm{d}}\right)\right]^{\frac{1}{2}}+2\left[\left(\gamma_{\mathrm{lj}}{ }^{\mathrm{p}}\right)\left(\gamma_{\mathrm{s}}{ }^{\mathrm{p}}\right)\right]^{\frac{1}{2}}$

The two components of surface free energy of the solid, $\gamma_{s}{ }^{d}$ and $\gamma_{s}^{p}$, can be determined by solving equation (10) and (11). The sum of these two quantities eventually gives the total surface energy of the solid.

\section{Application of the model}

Table 1. shows the water contact angle and surface energy of some commonly used polymers calculated from the Fowke'e extended model. The data corresponding to the first four rows in the table were obtained from literature [7] whereas the data in the last two rows were determined by the author $[3,8]$. It is interesting to note that the polar component of surface energy is very small in comparision to the dispersion component of the surface energy of the soilds taken from the literature. In contrast, polar component of surface enrgy is larger than the dispersion component for the polymer samples in our result. The difference in the proportion of polar and dispersion components may be due to the selection of test liquids. It has been recommended that one of the test liquid should be polar and the other non-polar for the accuracy of the determination of the components of surface energy. But due to the unavailability of purely non-polar liquid, we had used water and glycerol in our experiment both of which are polar liquids and this may have caused the error in the proportion of polar and dispersion components of the surface energies. However, the total surafce energy is in good agreement with the results reported in literature.

Table 1. Surface energy and water contact angle of some common polymers: ${ }^{1}$ obtained from the Literature and ${ }^{2}$ determined by the author.

\begin{tabular}{|l|c|c|c|c|}
\hline \multicolumn{1}{|c|}{ Material } & $\begin{array}{c}\text { Water } \\
\text { Contact } \\
\text { Angle }\left(\theta_{\mathrm{w}}\right)\end{array}$ & $\begin{array}{c}\text { Surface } \\
\text { Energy }(\gamma) \\
{\left[\mathrm{mJ} / \mathrm{m}^{2}\right]}\end{array}$ & $\begin{array}{c}\text { Polar } \\
\text { Component } \\
\left(\gamma^{\mathrm{p}}\right)\end{array}$ & $\begin{array}{c}\text { Dispersion } \\
\text { Component }\left(\gamma^{\mathrm{d}}\right)\end{array}$ \\
\hline Polyestyrene $^{1}$ & $91^{\circ}$ & 42 & 0.6 & 41.4 \\
\hline${\text { Nylon } 6-6^{1}}^{\circ}$ & $70^{\circ}$ & 43.2 & 9.1 & 34.1 \\
\hline PTFE $^{1}$ & $88^{\circ}$ & 14 & 1.5 & 12.5 \\
\hline Paraffin $^{1}$ & $66^{\circ}$ & 25.4 & 0 & 25.4 \\
\hline Polycarbonate $^{2}$ & $70^{\circ}$ & 35 & 18 & 17 \\
\hline Polyethylene $^{2}$ & $90^{\circ}$ & 20 & 15 & 5 \\
\hline
\end{tabular}

\section{Conclusion}

A brief introduction to the theory of contact angle analysis and subsequent calculation of surface energy from two liquid model has been presented. The approach used in our present paper is based on geometric mean of the surface energy components. The method was applied to determine the surface energy of polycarbonate and low density polyethylene by conntact angle measurement with water and glycerol. Our future work will be extended to the use of three liquid model for the better understanding of the surface energy. 


\section{Acknowledgement}

The author would like to thank Prof. Dr. Jan Janca and Dr. Lenka Zajickova, Department of Physical Electronics, Faculty of Science, Masaryk University, Czech Republic, for their valuable guidance during the course of my study.

\section{References}

D. K. Owens and R.C. Wendt, 1969. J. Appl. Polym. Sci. 13: 17411747.

D. P. Subedi, D. K. Madhup, A. K. Laghu, U. M Joshi and R. B. Tyata, 2008. Hydrophilization of low density polyethylene by dielectric barrier dischargeJournal of Science and Technology in the Tropics, 4, pp 73-77.

D. P. Subedi, 2002. Surface Modification of Polymers by Low Temperature Plasmas. 61137, Brno. Czech Republic.
D.Y. Kwok, C.N.C. Lam, A. Li, A. Leung, R.Wu, E. Mok and A.W. Neumann, 1998. Colloids and Surfaces A: Physicochemical and Engineering Aspects, 142, 219.

F. M. Fowke's Ind. 1964. Eng. Chem. 56 (40).

I. Sutherland and R. J. Heath, 1998. Application of contact angle measurement to wettability, surface energy and adhesion properties of polymers, Progress in Rubber and Plastic Technology, 14 (3): 151-173.

J. C. Vickerman, 1997. Editor, Surface Analysis, The Principal Techniques, John Wiley and Sons.

J. Kloubek, 1992. Developments of method for surface free energy determination using contact angles of liquids on solids, Advances on Colloid and Interface Science, 99-142.

Ph.D. thesis, Department of Physical Electronics. Masaryk University, Kotl'a`rsk'a 2. 